\title{
Spatial distribution characteristics of phaeozem in Songliao Plain based on RS
}

\author{
XING Yu*, Wang Jie, Yin Yaqiu, Zhou Yingjie, Yao Weiling, Wang Haiqing \\ China Aero Geophysical Survey and Remote Sensing Center for Land and Resources, Beijing 100083, China
}

\begin{abstract}
Phaeozem is one kind of scarce resources on the earth. Due to the restrictions of natural factors and the influence of human activities over the years, phaeozem degradation has become increasingly serious. Based on images of the ASTER in 2006 and the $\mathrm{ETM}^{+}$in 2000 in this paper, the phaeozem organic matter content which is greater than $2 \%$ was obtained by using quantitative retrieval. Through the three-dimensional remote sensing image, terrain rendering and relevant information were analyzed. On the basis of the above, the spatial distribution characteristics of phaeozem in Songliao Plain were analyzed using DEM (digital elevation model) data. The results show that the total area of phaeozem in Songliao Plain is about $51360.15 \mathrm{~km}^{2}$, and the phaeozem organic matter content is gradually increasing along with the increasing latitude or longitude, and decreasing along with increasing slope. In addition, the soil organic matter content is lower in southern slopes than that in northern slopes.
\end{abstract}

\section{Introduction}

Phaeozem is one of the most precious non-renewable scarce resources. Currently the only three phaeozem belts remaining are scattered in Ukraine, the Mississippi Valley, and Songliao Plain. With high organic matter content and sufficient nutrition supply, phaeozem is suitable for crop growth, and thus becomes the significant guarantee for the crop yield, which is undoubtedly an important strategic resource for a populous country where the per capita cultivated land only accounts for $43 \%$ of the world average. Since 1960s, land reclamation of the phaeozem has been rendered in a large scale and human activities has been enhanced, in addition to the influence of the natural environment and geological factors, the agro ecological environment in this area has been destroyed (for example, [1]) and the quality and quantity of the phaeozem is on the decline as well, which greatly threatens the local agricultural production, food security and the sustainable development of the society. As a result, a comprehensive knowledge of the phaeozem belt distribution of Songliao Plain for a constant supervision to the phaeozem erosion and deterioration becomes extremely urgent. Some scholars put emphasis on the study of the physical properties, the nutrient characteristics, causes of erosion, geochemical environment evaluation of phaeozem in scattered areas (for example, [2-4]), while quantity studies on the large scale spatial distribution in terms of the whole phaeozem belt are rare. Boasting such characters as high efficiency, great speed and dynamics, remote sensing technology (RS) has become one of the most effective way to conduct investigation for ecological condition. Based on RS data of the ASTER in 2006 and the $\mathrm{ETM}^{+}$in 2000, in combination with DEM data, this paper gives an quantitative analysis of the spatial distribution characteristics of the phaeozem, aiming at providing reliable data for the protection and sustainable utilization of phaeozem.

\section{Study area and phaeozem definition}

\subsection{Study area}

Songliao Plain is located in the interior of northeast China. The average annual temperature of Songliao Plain is 0.5 to $6^{\circ} \mathrm{C}$, and it has a warm and humid summer but a long and cold winter. The mean annual precipitation is 450 to $650 \mathrm{~mm}$, but the distribution is unbalanced. The rainfall from July to September accounts for more than half of the total precipitation, while the snowfall amount is little in winter, and seasonal frozen layer is common here. The frozen depth of the soil can reach 1.5 to $2 \mathrm{~m}$, and it can last for 120 to 200 days. The natural vegetation is miscellaneous meadow in the forest, which is locally called "tessellated meadow". Relying the phaeozem as the main cultivated soil, Songliao Plain not only shoulders the responsibility to sell 1500 million $\mathrm{kg}$ commodity grain to the state, but also acts as the main production area where sugar beet, flax, sunflowers and beans are planted.

\subsection{Phaeozem definition}

Under the steppe meadow vegetation in humid and semi-humid area, phaeozem is rich in deep humus accumulation and leaching processes and organic matter

\footnotetext{
* Corresponding author: xingyurs@163.com
} 
content. It has no reaction of lime in the whole profile with $\mathrm{pH} 6.5$ to 7.0 neutral reaction. The humus content in natural soil is $50 \mathrm{~g} / \mathrm{kg}$ to $80 \mathrm{~g} / \mathrm{kg}$, and cultivated soil $20 \mathrm{~g} / \mathrm{kg}$ to $40 \mathrm{~g} / \mathrm{kg}$, which has a tongue-like downward transition, and its base saturation is more than $70 \%$ (for example, [5]). According to the "standard for classification and gradation of soil erosion" (SL190-96), based on the definition and description for the phaeozem in northeast China in the sub-type of the national soil erosion level division that "take morphological principles (geology, landform and soil)as the basis", we can define the phaeozem region of northeast China as Da and Xiao Xing'an mountains, Changbai Mountain and its surrounding area, Sanjiang Plain, Songhua River and Liaohe alluvial plain as well(Horqin Sandy land is not included) .

\section{Method}

\subsection{General idea}

Based on images of the ASTER in 2006 and the ETM ${ }^{+}$in 2000 , the phaeozem organic matter content which is greater than $2 \%$ was obtained by using quantitative retrieval. Through the three-dimensional remote sensing image, terrain rendering and relevant information were analyzed. The spatial distribution characteristics of phaeozem in Songliao Plain were analyzed using DEM data. This thesis also gives a brief review of the methodology and technique of the estimation.

\subsection{Phaeozem information extraction}

Based on the 1:500000 phaeozem distribution graph provided by Nanjing Soil Institute, China Academy of Science, this paper works out the sampling line and takes advantage of GPS to record the longitude and latitude of the samples. The 80 samples chosen in May, 2006 and 2007, were sent to professional institutions to be detected in order to get the organic content of the phaeozem. The ASTER of the bare soil before spring plowing in 2006 is made atmospheric radiation correction through the model of FLAASH, and its apparent reflectivity ratio is calculated through the ENVI software(Formula 1). Multitemporal images are used in this research. To weaken the influence of such factors as atmosphere on spectral reflectance of the surface futures, the images data of the other months are corrected radiation with RS images in April as the base using ground "quasi" invariant feature points and statistical regression method (for example, [6]). The apparent reflectivity ratio $(\rho)$ is calculated as follows:

$$
\rho=\frac{\pi \times \text { gain } \times(\mathrm{DN}-1)}{E \times \sin (\theta)}
$$

In the equation gain is the sensor gain, $D N$ is the image brightness values, $E$ is solar irradiance, and $\theta$ is solar elevation angle.

To increase the accuracy of the phaeozem extraction, this research chooses the samples from waters and building land and makes an analysis of the reflectance spectral characteristics of those surface features in remote sensing images to find out the typical spectral characteristics and diagnosis band of all kinds of ground objects. Taken as the judging condition, the spectral data are input into the binary tree to mask off the waters and building land existed in the images.

There is a great relevance between the organic matter and the spectral reflectance of the phaeozem (for example, [7]). And the analysis of spectral features shows that this kind of relevance of the 9 wavebands of the ASTER data and the organic matter of the phaeozem reaches more than -0.5 , and the highest reaches -0.7 . Taking the soil spectral reflectance ratio as the independent variable, and the organic matter content as the dependent variable, we can formulate the equation of the linear regression on ASTER data (for example, [8-10]).

$\ln (S O M)=-7.531-1.268 \times \ln \rho 3-9.806 \times \ln \rho 9+$ $7.734 \times \ln \rho 8+0.653 \times((\rho 4-\rho 2) / 0.995)$

In the equation $\rho i$ is the apparent reflectivity ratio of band $i(i=1,2, \ldots, 9) ; S O M$ is the soil organic matter content. The decision coefficient of the model is 0.851 , and the total root mean square difference is 0.24 .

The humus content in natural phaeozem is $50 \mathrm{~g} / \mathrm{kg}$ to $80 \mathrm{~g} / \mathrm{kg}$, and in cultivated phaeozem is $20 \mathrm{~g} / \mathrm{kg}$ to $40 \mathrm{~g} / \mathrm{kg}$. The soil which has more than $20 \mathrm{~g} / \mathrm{kg}$ organic matter content is extracted as the phaeozem in this research.

RS image processing may destroy the original spectral information, and makes an impact on quantitative inversion of remote sensing. Therefore, this study will take advantage of the original data to make an inversion calculation, and then the organic content map of the soil worked out from the inversion calculation will be made a geometric correction. Now is the detailed process. Taking a 1:100000 topographic map as the reference images, and the ASTER images as the matching map, to make a correction, we can get the ground control points (GCP) of the ASTER, which will be made use of to correct the results of inversion calculation. The error could be limited in one pixel.

\subsection{DEM analysis}

Techniques like multistage pyramid and elevation zone makes it possible that the three dimensional terrain visualization and 3D RS image processing are accessible to us, which does not only reflect the rich RS information, but also vividly presents the topography and its related information. And all of those play a significant role in phaeozem interpretation. After the correction and mosaic for the ETM images for Songliao Plain, we can work out a DOM, which acts as the texture to map on the DEM, and the dimensional RS images come out. In addition, the multistage pyramid is added to the surface of DOM so that the dimensional RS images displayed speed could be enhanced. To get a better visual effect and get rid of the disturbance between colors, a elevation zoning render graph of Songliao Plain will be formed.

Using the ideology of intersection analysis after MASK handled, the vector data of phaeozem is converted to the grid data. The elevation of phaeozem is 
automatically acquired by intersection analysis, after a small amount of DEM come out by MASK technology. And then multiple terrain parameters are calculated and analyzed based on DEM (for example, [11]), so that we obtain the relationship between the spatial distribution of phaeozem and terrain in Songliao Plain.

\section{Results and analysis}

\subsection{Distribution characteristics}

The research results shows the phaeozem belt stretches from the Heihe City, in Heilongjiang Province in the north, to Changtu, in Liaoning Province in the south. And in the west, it begins from Gannan County in Qiqihar where it's bounded by Songliao Plain which is in chernozem and saline-alkali soil, to Bin County in the middle of Harbin in the east. The phaeozem belt is a meniscus shaped distribution along the railway line from Harbin to Siping and Bei'an, in the middle reaches of Nenjiang river and the west side of Xiaoxing'anling. The total area of phaeozem is $51360.15 \mathrm{~km}^{2}$ in Songliao Plain.

The study also finds out that the organic matter content in phaeozem is distributed in a tongue-like downward transition. The organic matter content is highest in Wudalianchi City, Heilongjiang Province, and next is in Beian City, and the organic matter content in both cities reaches above $6 \%$. From Hailun County to Suiling County and Baiquan County to Mingshui County, the soil organic matter content in the phaeozem presents a decreasing trend, and from Baiquan County to Hailun County, and Mingshui to Suiling County, the soil organic matter content is on the rise. The organic matter content of phaeozem in Baiquan County, Mingshui County, Qinggang County and Lanxi County decreases gradually. From Suihua City where the organic matter content is $4 \%$, to Harbin City, the soil organic matter content reduces gradually, whereas it is significantly higher in Shuangcheng City than in surrounding areas. With an average organic matter content of $3 \%$, the content is lower in Jilin Province than in Helongjiang Province on the whole. The most southern part of the phaeozem belt in Songliao Plain which is located in Liaoning Province, is not that typical. With the organic matter content of phaeozem in Liaoning Province lower than the average content of the phaeozem, the color and hue in the RS images which represents the phaeozem in Liaoning Province is shallower than that in Helongjiang and Jilin Province.

The organic matter content in the west part of the phaeozem belt in Songliao Plain is obviously lower than that in the east and shows a trend of gradual increase with the increase of longitude; and the organic matter content increases gradually from south to north, with a rising trend accompanied by the increase of latitude. The phaeozem in Wudalianchi City which lies in the most northeast corner enjoys the highest organic matter content, while it is the lowest in Changtu County, Tieling City in the southwest corner. The phaeozem organic matter content in north of Suihua City is almost above $4 \%$, which is comparatively high, while in south of Suihua, it's basically below 4\%. According to the definition of phaeozem and its related materials, the phaeozem organic matter content should be more than $2 \%$. Based on the inversion chart of soil organic matter content and chernozem data, the organic matter content in the northwest part of Mingshui County, from Yilong County in southern of Yi'an County to Zhongxin County, and Xintun area in northwestern of Yi'an County, is comparatively higher, but these high value zone of organic matter content belongs to chernozem.

\subsection{Terrain characteristics}

The terrain of the phaeozem area is mainly upland plain, platforms and terrace formed by cutting of different degree from new tectonic movement. The slope and aspect of the phaeozem terrain are calculated based on DEM data. We find out the slope range of the phaeozem zone is $0^{\circ} \sim 2^{\circ}$, the average slope is $1.69^{\circ}$ and the average aspect is $175.14^{\circ}$. Superimpose the RS images of the whole phaeozem area and the elevation zone with DEM, and work out three dimensional remote sensing images and elevation zone render graph with the help of software, which will provide reliable topographic data for the study of spatial distribution of the phaeozem.

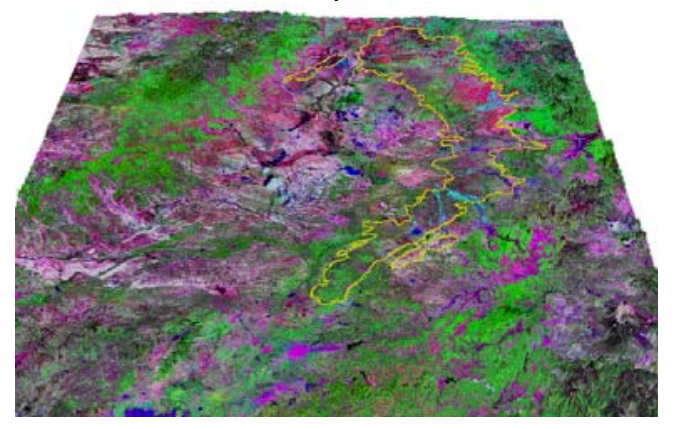

Fig. 1. Three-dimensional remote sensing image and phaeozem area in Songliao Plain

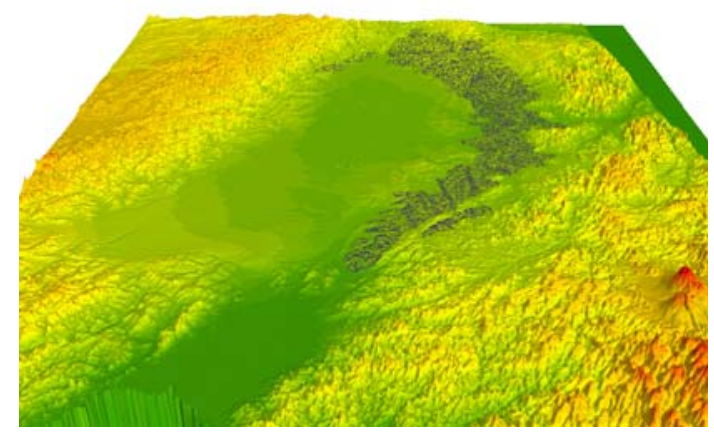

Fig. 2. Rendering of phaeozem terrain in Songliao.Plain

Through the analysis, we can find out the organic matter content of the phaeozem in east and west slope of this area is relatively low, which is associated with the geomorphological characteristics of the study area. Hills in this area mostly show a north-south orientation. The north and south slope are relatively flat, while the west and east slope are steep and the soil erosion is serious. The phaeozem organic matter content of the sunny slopes is 
lower than that of the shady slopes, which is presented by the fact that the organic matter content of the south slope is lower than that of north slope, and southeast slope lower than northeast slope. The solar radiation received by sunny and shady slopes is different, which leads to different water and heat conditions and microclimate. And all the above make an influence on the vegetation growth situation and the organic matter content eventually. According to the related researches made by domestic and foreign scholars on freeze-thaw erosion (for example, [12]), the sunny slope receives more solar radiation, and the temperature difference between day and night in sunny slope is greater than that in shady slope, and then the freezing and thawing is stronger in sunny slope. In addition, the snow of the sunny slope melts faster in spring, and the water is relatively concentrated, which makes the soil erosion more serious in sunny slope.

It shows a trend that the steeper the slope is, the lower the phaeozem organic matter content is in the study area. The study finds that the organic matter content of $0^{\circ} \sim 1^{\circ}$ slope is significantly higher than that of other slopes. We can owe this phenomenon to the fact that with the slope increasing, there is less time for the rainfall to infiltration, and the rainfall runoff goes faster, which speeds up the rate of soil erosion and then exacerbate the loss of phaeozem organic matter. The phaeozem organic matter content of similar slope position is almost the same.

\section{Conclusion}

Through the research, we can get the conclusions as follows: Songliao Plain boasts a total phaeozem area of $51360.15 \mathrm{~km}^{2}$, which is a meniscus shaped distribution between the Heihe City, to Changtu. And in the west, it's bounded by Songliao Plain which is in chernozem and saline-alkali soil, to Bin County in the middle of Harbin in the east. It shows the trend that the phaeozem organic matter content increases gradually with the increase of longitude or latitude; and the overall organic matter content of phaeozem of the sunny slopes is lower than that of the shady slopes. It also shows the trend that the steeper the slope is, the lower the phaeozem organic matter content is; and the phaeozem organic matter content of similar slope position is almost the same. It is clear that the phaeozem spatial distribution features in our country will provide foundational data for the phaeozem management and regional sustainable development.

\section{Acknowledgements}

This paper is supported by Programs of China Geological Survey (DD20201180, DD20190705, DD20190511). Thank for guidance and help in the work and writing process from Jiang Qigang, Li Yuanhua and other experts.

\section{References}

1. Sui Y Y, Zhang X Y, Zhang S L, et al, 2008, Soil organic matter actuality of the black soil farmland in
Heilongjiang counties, Chinese Journal of Soil Science, Vol. 39, No. 1, pp. 186-188.

2. Hill J, Schiitt B, 2000, Mapping complex patterns of erosion and stability in dry Mediterranean ecosystems, Remote Sensing of Environment, Vol. 74, pp. 57-569.

3. Fox G A, Sabbagh G J, 2004, An automated soil line identification routine for remotely sensed images, Soil Science Society of America Journal, Vol. 68, pp. 1326-1331.

4. Fox G A, Sabbagh G J, 2002, Estimation of soil organic matter from red and near-infrared remotely sensed data using a soil line Euclidean distance technique, Soil Science Society of America Journal, Vol. 66, pp. 1922-1929.

5. Institute of Forestry and Soil Science, Chinese Academy of Science, 1980, Soil in the northeast of China, Science Press, Beijing.

6. Guo J N, Yu J, Zeng Y, et al, 2005, Study on the relative radiometric correction of CBERS satellite CCD image, Science in China, Vol. 48, pp. 12-28.

7. He T, Wang J, Lin Z J, et al, 2006, Spectral features of soil organic matter, Geometrics and Information Science of Wuhan University, Vol. 31, No. 11, pp. 975-978.

8. Cheng B, Jiang Q G, 2008, Estimation of soil properties using remotely sensed imagery, Chinese Agricultural Science Bulletin, Vol. 24, No. 1, pp. 467-470.

9. Fang C S, Lu L S, Zhong Y H, et al, 2007, The Source Analysis of the Components in Total Suspended Particulates in Ambient Air of Typical Cities in Jilin Province, Journal of Jilin University (Earth Science Edition), Vol. 37, No. 5, pp. 988-992.

10. Xing $\mathrm{Y}$, Jiang $\mathrm{Q} \mathrm{G}$, Li $\mathrm{Y} \mathrm{H}$, et al. Extracting phaeozem information in Songliao Plain based on remote sensing and GIS[J]. Transactions of the CSAE, 2010, 26(10):212-217.

11. Dai Y Q, Ren J W, Shen X H, 2009, Precision estimation and geomorphological analysis based on the DEM generated by InSAR: Taking Damxung-Yangbajain area as an example, Earthquake Science, Vol. 22, pp. 263-269.

12. Zhang J G, Liu S Z, Yang S Q, et al, 2007, The classification and assessment of freeze-thaw erosion in Tibet, Journal of Geographical Sciences, No.2, pp. 165-174. 